\title{
The Space Density of Galaxy Peaks and the Linear Matter Power Spectrum.
}

\author{
Rupert A.C. Croft $^{1}$ \\ Department of Astronomy, The Ohio State University, Columbus, Ohio 43210, USA \\ Enrique Gaztañaga ${ }^{2}$ \\ CSIC, Institut d'Estudis Espacials de Catalunya (IEEC), Edifici Nexus-104 - c/ Gran Capitan \\ 2-4, 08034 Barcelona, Spain
}

\begin{abstract}
One way of recovering information about the initial conditions of the Universe is by measuring features of the cosmological density field which are preserved during gravitational evolution and galaxy formation. In this paper we study the total number density of peaks in a (galaxy) point distribution smoothed with a filter, evaluating its usefulness as a means of inferring the shape of the initial (matter) power spectrum. We find that in numerical simulations which start from Gaussian initial conditions, the peak density follows well that predicted by the theory of Gaussian density fields, even on scales where the clustering is mildly non-linear. For smaller filter scales, $r \lesssim 4-6 h^{-1} \mathrm{Mpc}$, we see evidence of merging as the peak density decreases with time. On larger scales, the peak density is independent of time. One might also expect it to be fairly robust with respect to variations in biasing, i.e. the way galaxies trace mass fluctuations. We find that this is the case when we apply various biasing prescriptions to the matter distribution in simulations. If the initial conditions are Gaussian, it is possible to use the peak density measured from the evolved field to reconstruct the shape of the initial power spectrum. We describe a stable method for doing this and apply it to several biased and unbiased non-linear simulations. We are able to recover the slope of the linear matter power spectrum on scales $k \lesssim 0.4 h^{-1} \mathrm{Mpc}^{-1}$. The reconstruction has the advantage of being independent of the cosmological parameters $\left(\Omega, \Lambda, H_{0}\right)$ and of the clustering normalisation $\left(\sigma_{8}\right)$. The peak density and reconstructed power spectrum slope therefore promise to be powerful discriminators between popular cosmological scenarios.
\end{abstract}

Subject headings: Large-scale structure of the universe - galaxies: clustering, methods: numerical, statistical

\footnotetext{
${ }^{1}$ CSIC, Institut d'Estudis Espacials de Catalunya (IEEC), Edifici Nexus-104 - c/ Gran Capitan 2-4, 08034 Barcelona, Spain

${ }^{2}$ Oxford University, Astrophysics, Keble Road, Oxford OX1 3RH, UK
} 


\section{Introduction}

Attempts to reconstruct the initial conditions from which large-scale structure in the Universe grew have been carried out using several different methods. One avenue of research involves attempting to "run gravity backwards" from the present day galaxy distribution. This has been done with dynamical schemes (eg. Peebles 1989, Nusser \& Dekel 1992, Croft \& Gaztañaga 1997) and also by applying a one to one mapping between the final smoothed density and the initial density (assumed to have a Gaussian p.d.f.) (Weinberg 1992). A different approach attempts to recover statistical measures of the initial conditions (eg. the two-point correlation function or the power spectrum) from knowledge of how gravitational evolution has affected these statistics. For example, formulae have been proposed which map the correlation function measured at the present day onto the initial correlation function (Hamilton et al. 1991). The same is true for the power spectrum (Peacock and Dodds 1994, Jain, Mo \& White 1995, Baugh \& Gaztañaga 1996). In this paper we will show how the initial power spectrum shape can be inferred from measurements of the space density of peaks in the galaxy distribution, even on scales where significant non-linear evolution has taken place in the underlying mass.

Peaks in the initial density distribution have been thought to be potential sites for the formation of galaxies and clusters of galaxies. Two seminal papers by Kaiser (1984) and Bardeen et al. (1986) in which the details of the theory of Gaussian random fields relevant to these problems were studied have been very influential in the study of galaxy and large-scale structure formation. In the present paper we apply these results to the density field smoothed on larger scales, in order to see how peak theory describes the properties of an evolved density field. We are particularly interested in seeing whether the distribution of peaks enables us to recover some information about the initial conditions, and also in its potential use as a discriminator between cosmological models.

When applying peak theory to the problem of galaxy or cluster formation, the assumption is usually made that these objects have formed from the gravitational collapse of matter around a high peak. Some smoothing scale, somewhat arbitrarily chosen, is associated with the object to be formed. The validity of these assumptions has been tested on the small scales relevant to the formation of individual objects by Park (1991), and Katz et al. (1993) amongst others. The conclusions seem to be that at least for the formation of matter haloes, there is only qualitative agreement between the predictions and the results of numerical simulations. Indeed in view of the extensive merging of matter clumps predicted by presently favoured hierarchical models of structure formation (see e.g. Press \& Schecter 1974, Lacey \& Cole 1993) it is reasonable to expect that evolution of the density field on small scales will tend to disrupt the predictions of peak theory.

When the matter distribution is smoothed on somewhat larger scales however, for example in the estimation of the genus (e.g. Melott, Weinberg \& Gott 1988), the topology and some aspects of large scale features of the density field seem to be little affected by gravitational evolution. In particular, the rank order of density contrasts measured at points in the field is approximately 
conserved. This fact has been used by Weinberg (1992) in the reconstruction method mentioned above. This reconstruction method also appears to work well when tested on simulations where the matter distribution is biased using some ad hoc galaxy formation model.

In this paper we will use numerical simulations to test whether this insensitivity to gravitational evolution affects the mean number of peaks and if so on what scales it begins to break down. We are also interested in reconstructing the power spectrum shape from the peak density as one specifies the other entirely in Gaussian models. The outline of the paper is as follows: In Section 2 we will briefly introduce the concept of the number density of peaks in Gaussian random fields and in $N$-body simulations. We will describe the simulations and our method of peak selection before testing peak theory against the evolved density both of mass and of 'galaxies' identified in the models. In Section 3 (and Appendix A) we will describe a stable method of recovering the power spectrum slope from the peak density. We will test it first on

analytic power spectra and then on the simulation results. In Section 4 we discuss our results and present some conclusions.

\section{The number density of peaks}

We consider local maxima of all heights in a density field to be peaks. In this paper we will only consider cosmological models which start from Gaussian initial conditions. The derivation of quantities related to the power spectrum of fluctuations will rely on this assumption, but of course the raw peak density itself when measured in observations can be used to apply constraints to other sorts of models.

\subsection{Peaks in Gaussian random fields}

For a Gaussian random field the number density of peaks per unit volume $n_{p k}$ is given by (Bardeen et al. 1986):

$$
n_{p k}=\frac{29-6 \sqrt{6}}{2(2 \pi)^{2} 5^{3 / 2}} R_{*}^{-3},
$$

where $R_{*}$ is the ratio of moments of the power spectrum $P(k)$ corresponding to a characterstic scale in the Gaussian field:

$$
\begin{aligned}
R_{*} & \equiv \sqrt{3} \sigma_{1} / \sigma_{2}, \\
\sigma_{1}^{2} & \equiv \frac{1}{2 \pi^{2}} \int d k k^{4} P(k), \\
\sigma_{2}^{2} & \equiv \frac{1}{2 \pi^{2}} \int d k k^{6} P(k),
\end{aligned}
$$


In a practical situation the field has to be smoothed on the scale, $r_{f}$, of interest. We will use a Gaussian window filter, so that $P(k)$ in the above equations should be multiplied by $\exp \left[-\left(k r_{f}\right)^{2}\right]$.

\subsection{Peaks in numerical simulations}

To find maxima we need the density field to be differentiable, which means that a filter must be applied to the particle based realisation of density derived from $N$-body simulations. An additional limitation imposed on us by simulations is their limited ability to resolve underdense regions, which will largely affect minima. These are present in identical numbers to maxima in Gaussian fields. The sampling of maxima being in general better than that of minima (most local maxima are 'higher' than most local minima) we choose to study only maxima here.

We use simulations of three different spatially flat cold dark matter dominated Universes. One set of simulations is of "standard" CDM, with $\Omega_{0}=1, h=0.5$, and another is of low density CDM with $\Omega_{0}=0.2, h=1$ and a cosmological constant $\Lambda=0.8 \times 3 H_{0}^{2}$. The power spectra, $\mathrm{P}(\mathrm{k})$ for these models are taken from Bond \& Efstathiou (1984) and Efstathiou, Bond and White (1992). The shape of $\mathrm{P}(\mathrm{k})$ is parametrised by the parameter $\Gamma=\Omega h$, so that we have $\Gamma=0.5 \mathrm{CDM}$ and $\Gamma=0.2$ CDM. We also use simulations of a Mixed Dark Matter universe where a massive neutrino component contributes $\Omega_{\nu}=0.3, \Omega_{C D M}=0.6$ and $\Omega_{\text {baryon }}=0.1$. The power spectrum was taken from Klypin et al. (1993). Each simulation contains $10^{6}$ particles in a box of comoving side-length $300 h^{-1} \mathrm{Mpc}$ and was run using a $\mathrm{P}^{3} \mathrm{M} N$-body code (Hockney and Eastwood 1981, Efstathiou et al. 1985). The mean comoving interparticle separation is therefore $3 h^{-1} \mathrm{Mpc}$, of the same order as that of normal galaxies. The simulations are descibed in more detail in Dalton et al. (1994). In this paper we use 5 realisations of each model, error bars being estimated from the standard deviation of their results.

To find peaks, we first assign the particle density to a $256^{3}$ grid using a cloud in cell scheme ( Hockney \& Eastwood 1981). We then smooth the density field in Fourier space using a Gaussian filter of the appropriate radius. We then identify local maxima on the grid in real space. We carry out this procedure for several logarithmically spaced values of the filter radius. We find that if the filter radius is smaller than the mean interparticle separation the number of peaks increases, due to discreteness effects, as isolated particles become erroneously identified as peaks. The finite grid may also have a small effect in the opposite direction as we cannot identify peaks which are less than two grid cells apart. In this paper we choose to analyse fully sampled simulations, using filter radii from $3 h^{-1} \mathrm{Mpc}$ to $50 h^{-1} \mathrm{Mpc}$. We defer the study of discreteness effects, which will be important in the study of real galaxy surveys, to future work. In finding peaks, we also make use of the periodic boundary conditions in the simulations. The boundaries of real surveys will also have an effect which will need to be quantified.

Our results, the number density of peaks as a function of filter radius are shown in Figures 1 and 2 . When plotting our results, in order to make the figures clearer, we have chosen to plot 


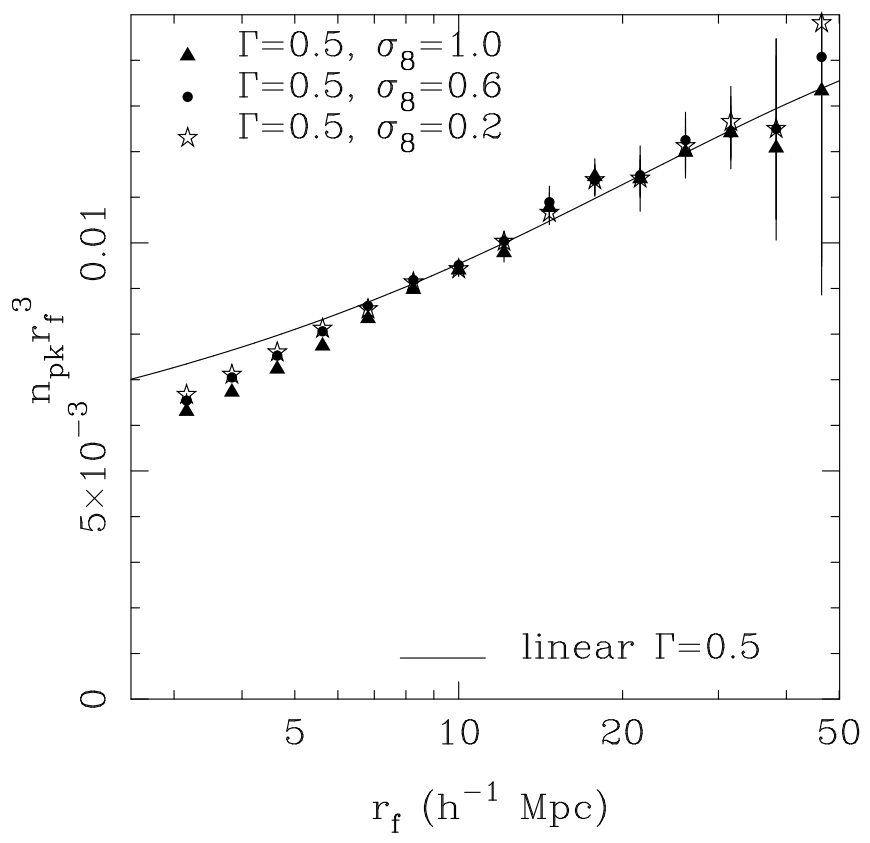

Fig. 1.- Peak space density (actually $n_{p k} r_{f}^{3}$, proportional to the mean number of peaks per smoothing volume) for three different output times in the Standard $(\Gamma=0.5) \mathrm{CDM}$ scenario. The errors on the points are calculated from the standard deviation about the mean of results from 5 simulations. The linear theory prediction is shown by a curve.

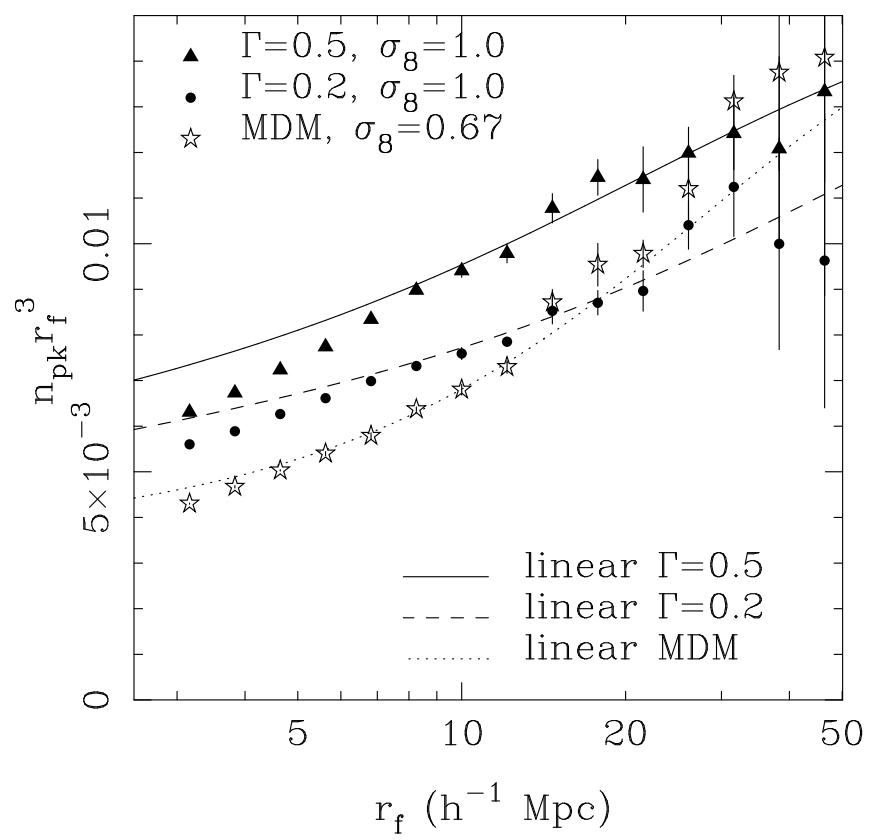

Fig. 2. $-n_{p k} r_{f}^{3}$ for three different cosmological models, $\Gamma=0.5 \mathrm{CDM}, \Gamma=0.2 \mathrm{CDM}$, and Mixed Dark Matter The linear theory predictions are shown by curves. 
not the peak space density but $n_{p k}$ multiplied by $r_{f}^{3}$. This is proportional to the number of peaks enclosed on average by a smoothing volume. We show results for the $\Gamma=0.5 \mathrm{CDM}$ simulation at different stages in its evolution (Figure 1), as well as results for the other models (Figure 2). The simulation points are labelled according to the predicted linear theory amplitude of fluctuations in $8 h^{-1} \mathrm{Mpc}$ radius spheres, $\sigma_{8}$. The models shown in Figure 2 have a fluctuation amplitude in the range allowed by the 4 year COBE results (Hinshaw et al. 1996). In the figures, we also show as curves the predictions of peak theory of Gaussian random fields calculated using the formulae of Section 2.1 .

During the course of gravitational evolution, we do expect the peaks to move and to change in height through accretion of matter. Indeed, the number density of objects above a certain mass threshold changes rapidly, and can be used as a sensitive probe of the value of $\sigma_{8}$ (see eg. White, Efstathiou and Frenk 1993). However, in this paper we are interested in the total space density of peaks of all heights, which is predicted to be constant in time in linear theory.

We can see from the first two figures that the measured number of peaks follows the theoretical prediction remarkably well, particularily on filter scales $r_{f} \gtrsim 4-6 h^{-1} \mathrm{Mpc}$. We attribute to merging the slight decrease in the number of peaks over time which we see in Figure 1. Of the two CDM models, the $\Gamma=0.5$ model, which has relatively more power on small scales departs the most from the prediction. Merging is not a very important process, though, as even on scales of $3 h^{-1} \mathrm{Mpc}$, the space density of peaks only falls $\sim 10-15 \%$ below the peak theory prediction by the time the simulation has reached $\sigma_{8}=1$. There appears to be even less merging in the MDM model.

The amplitude of fluctuations in the density field smoothed on the scales where the peak theory prediction begins to break down is $\langle\delta>\sim 1-2$, which is in what is conventionally referred to as the quasilinear regime. We are therefore probing roughly the same scales where higher order perturbation theory (eg. Baugh, and Efstathiou 1994) can be used to estimate departures from linear theory.

\subsection{Biased simulations}

The relationship between galaxies and mass is one of the great uncertainties which affects the study of large-scale structure. It would be very useful to be able to measure properties of the galaxy density field and know exactly how they are related to those of the underlying mass. In the calculation of statistical clustering measures such as the two-point correlation function the uncertainty is parametrised with a linear biasing parameter. The value of this parameter in the real Universe, is still an unknown, if indeed it can be measured at all. In the case of the peak density, we can be hopeful that differing relationships between galaxies and the mass will have a smaller effect than on the two-point correlation function. It seems reasonable to expect that where a concentration of matter forms a peak in the density field there will also be a peak of some sort 


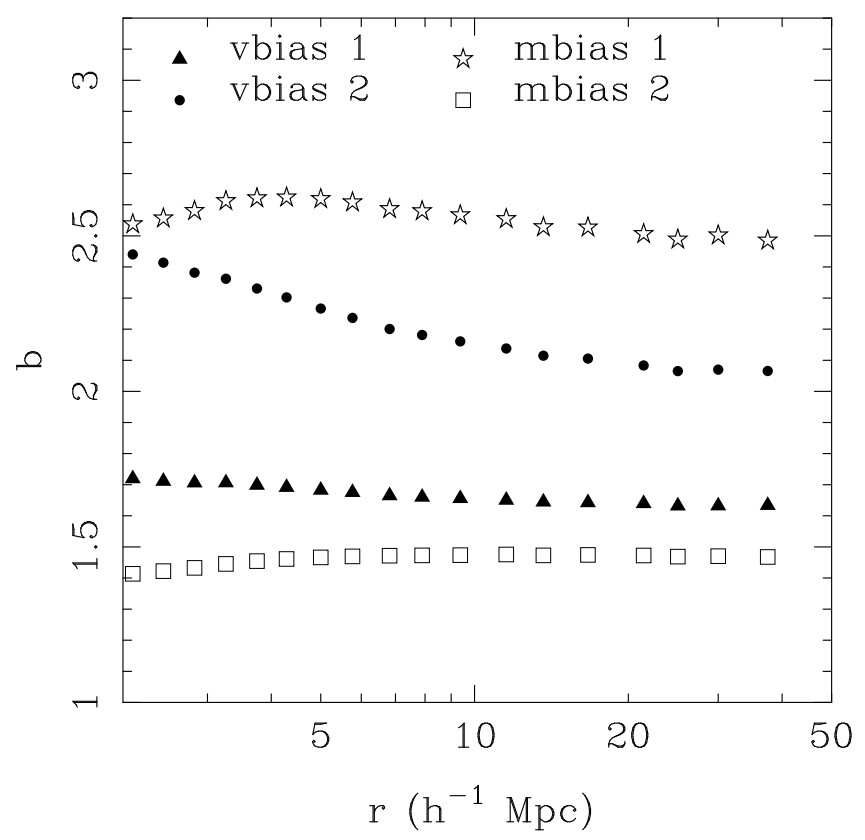

Fig. 3.- The effective bias $b \equiv \sigma_{\text {galaxies }}(r) / \sigma_{\text {mass }}(r)$ as a function of scale for 4 different biasing prescriptions applied to the $\Gamma=0.5$ simulations (see Section 2.3).

in the galaxy distribution. In this case, when we measure the total space density of peaks in the galaxy distribution we are measuring the equivalent quantity for the mass. Of course it is possible to imagine that no correlation exists between the galaxies and mass, or that the relationship is extremely complex and very non-local, so that our assumptions do not hold. Fortunately, there is some evidence that this is not the case, at least on large scales. For example, there appears to be a good correlation between galaxy velocities predicted from the density field and the actually measured velocities, which respond to the underlying mass (see eg. da Costa et al. 1997).

In order to partially test these ideas, we have applied different ad hoc biasing prescriptions to the $\Gamma=0.5$ simulation in order to produce a set of galaxy particles. One prescription involves carrying out the following procedure. For each particle we find the distance to the 20th nearest particle and then calculate the overdensity inside a sphere of this radius. If the resulting fluctuation is above a certain critical value (in this case 0.5) we add the particle to the list of galaxies. This has been done for the $\Gamma=0.5 \mathrm{CDM}$ simulation, for outputs with linear theory mass $\sigma_{8}=0.5$ and $\sigma_{8}=0.67$. As the prescriptions are based on the overdensity of a given mass of particles, we label the resulting results "mbias 1" and "mbias 2" respectively. For the "mbias 2" prescription, we also add a random $30 \%$ of "field" particles to the list of galaxies, to represent peaks which could have formed in lower density regions but did not due to the finite resolution of the simulation. The second prescription involves finding the overdensity in a fixed volume about each particle (in this case a sphere of radius $2 h^{-1} \mathrm{Mpc}$ ). We add the particle to the list of galaxies if the overdensity is above 1.0 for one prescription or 3.0 for another. We label these sets "vbias 1 " and "vbias 2" respectively. The $\Gamma=0.5 \mathrm{CDM}$ simulation with $\sigma_{8}=0.5$ was used. 


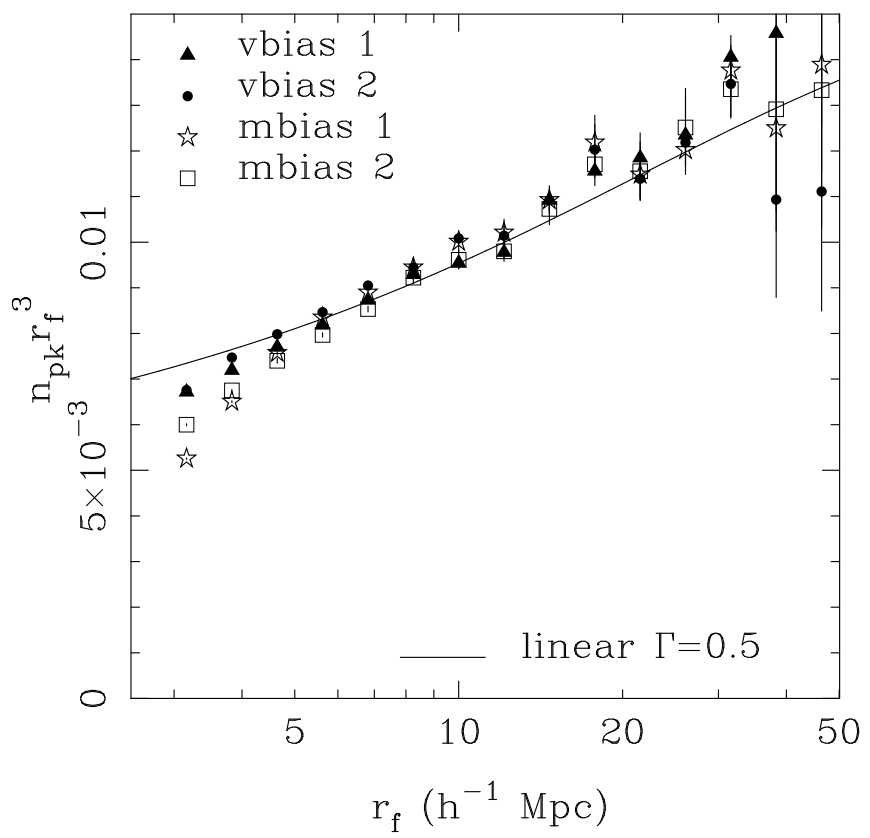

Fig. 4.- $n_{p k} r_{r}^{3}$ for the 4 different galaxy biasing prescriptions (see Section 2.3 for details) applied to simulations of the Standard $(\Gamma=0.5) \mathrm{CDM}$ scenario.

In all these cases, galaxy particles are preferentially selected in high density regions and are more clustered than the underlying mass. In Figure (3) we show how the bias factor $\sigma(r)_{\text {galaxies }} / \sigma(r)_{\text {mass }}$ varies as a function of scale. We can see that all the models have some weak scale dependence on small scales, with the mbias 1 galaxies having the largest bias factor on all scales.

The effect of these biasing prescriptions on the peak space density can be seen in Figure 4, where we also plot the linear theory prediction for the $\Gamma=0.5 \mathrm{CDM}$ model. For filter scales above $\sim 6 h^{-1} \mathrm{Mpc}$, the results are in good agreement with linear theory, as was the case for the unbiased simulations. Below this, we see a deficit in the number of peaks, which is greatest for the mbias 1 and mbias 2 prescriptions, in which galaxies are selected according to the overdensity of a given number of surrounding particles and not governed by a physical length scale. For all biasing prescriptions, the deficit of peaks on small scales is something we should expect, as when we choose galaxies, we are effectively picking out peaks above a certain height in the evolved mass distribution. The peaks that fall below the threshold will disappear. The effect does not seem to be too drastic though, and on all but the smallest scales is smaller than the differences between cosmological models (see Figure 2). 


\section{Reconstruction of the power spectrum slope}

All the models we have tested in Section 2 started from initial conditions with Gaussian random phases. In these cases, all statistical information about the initial density is contained in the power spectrum, $P(k)$. From Equations 1 and 2, we can see that the shape (but not the amplitude) of the linear power spectrum is responsible for the space density of peaks in the initial density field. In this Section we will present a simple and stable method for inverting these relations and recovering the shape of $P(k)$ from the space density of peaks. We will then apply this method to the space density of peaks measured from simulations. Since Section 2 has shown us that on quasilinear scales this is the same as $n_{p k}$ in the initial conditions, we will therefore be reconstructing the initial power spectrum shape.

Given the number density of peaks for a Gaussian field in equation (1), what can we say about its power spectrum? In other words, what information about $P(k)$ can be obtained from the ratio of its moments, $R_{*}$, in equations 2-3? It is clear from these equations that $R_{*}$ is independent of the amplitude of $P(k)$, but it depends on its shape. If we wanted to compare with a known family of power spectrum shapes (e.g. CDM), we could just find the best parameter in the family (e.g. $\Gamma)$ to fit the values of $R_{*}$ as a function of $r_{f}$. To find the power spectrum shape more generally, one could try using a numerical inversion technique (eg. a generalization of the method of Lucy 1974) to extract the shape of $P(k)$ from the measured values of $R_{*}$.

A simpler approach, which we adopt here, is to approximate $P(k)$ locally with a power law $P(k)=k^{n}:$

$$
n(k) \equiv \frac{d \log P(k)}{d k}
$$

If the slope $n(k)$ is constant with scale, then we substitute $P(k)=k^{n} \exp \left[-\left(k R_{f}\right)^{2}\right]$ into Equations 2 and 3 and find (Bardeen et al. 1986):

$$
R_{*}=\left(\frac{6}{n+5}\right)^{1 / 2} r_{f}
$$

This is only exactly true if $n$ is a constant. However, if we assume that $P(k)$ has a slowly varying shape, then it will remain a good approximation when applied locally. $n$ is then given by the local value at $k \sim 1 / r_{f}$, (where the Gaussian filter gives its maximum contribution). In Appendix A we show how to find the best effective relation between $k$ and $r_{f}$. We can then use Equation 4 to translate the estimated values of $R_{*}$ at a given $r_{f}$ to mean values of $n$ at a given $k$. In Appendix A, we also test the method with various different analytic power spectra, going from the power spectrum to the peak density and then back to the reconstructed power spectrum slope. We find that the method is stable and works well. 


\subsection{Reconstruction from the peak density in simulations}

When a density field undergoes non-linear evolution under gravity, mode coupling causes a relative transfer of power between large and small scales. In the cases considered here, this has the effect of making $P(k)$ less steep on small scales (see, eg. Baugh \& Efstathiou 1994). In Figure 9 we show $n(k)$, the logarithmic slope of $P(k)$ measured from the evolved mass distribution of our three different cosmological models. This was estimated by first calculating $P(k)$ using an FFT and then applying a two-point finite difference operator to recover $n$ at the points shown in the figure. We also plot the linear theory predictions for $n$, which enables us to see that non-linear evolution of $n$ is visible in the simulations on scales $k \gtrsim 0.07 h^{-1}$ Mpc. From Equation A1, using $\eta \simeq 0.4$, this scale is equivalent to $r_{f} \lesssim 20 h^{-1} \mathrm{Mpc}$.

We now apply our reconstruction method described in to the peak density measured from the simulations. This results in an estimate of $n$ as a function of scale which we plot in Figure 10. The reconstructed $n$ values are reasonably close to the linear theory values, as we would expect. Again, the $\Gamma=0.5 \mathrm{CDM}$ simulation suffers the most from non-linear effects. It is interesting that the non-linearities (ie. merging) which affect the peak density cause it to be lowered, which means that the recovered $n$ is slightly steeper than linear theory. We can see this on the smallest scales in Figure 11, in which we plot the reconstructed $n$ for different output times in the $\Gamma=0.5$ CDM simulation. This is opposite to the effect of non-linear evolution on the directly measured value of $n$ (see Figure 9).

In Figure 12 we show that the reconstruction also works well on the peak density measured from the biased simulations, and again works best on larger scales.

\section{Discussion and conclusions}

Although a local transformation of a density field can change the heights of peaks, it should not affect the peak number density. Thus, as far as the effects of both gravity and biasing are approximately local one should expect the galaxy peak density to be a preserved quantity. Gravity is local on linear scales and there has not been enough cosmic time for gravity to affect (or become non-linear on) scales much larger than $\sim 8 h^{-1} \mathrm{Mpc}$. For similar reasons it also seems difficult for biasing to be significantly non-local (see also Gaztañaga \& Frieman 1994 and references therein). Of course, it may also be possible to have non-local transformations which preserve the peak number density.

Even without attempting to reconstruct the power spectrum shape from it, the space density of peaks appears to be an interesting statistic. At the present time, the two cosmological models which are the most favoured alternatives to $\Gamma=0.5 \mathrm{CDM}$ are Low density CDM and MDM, which we have seen have very differently shaped linear power spectra and different peak space densities. The similarity of their shapes on quasilinear scales, and the uncertainties from biasing on smaller 


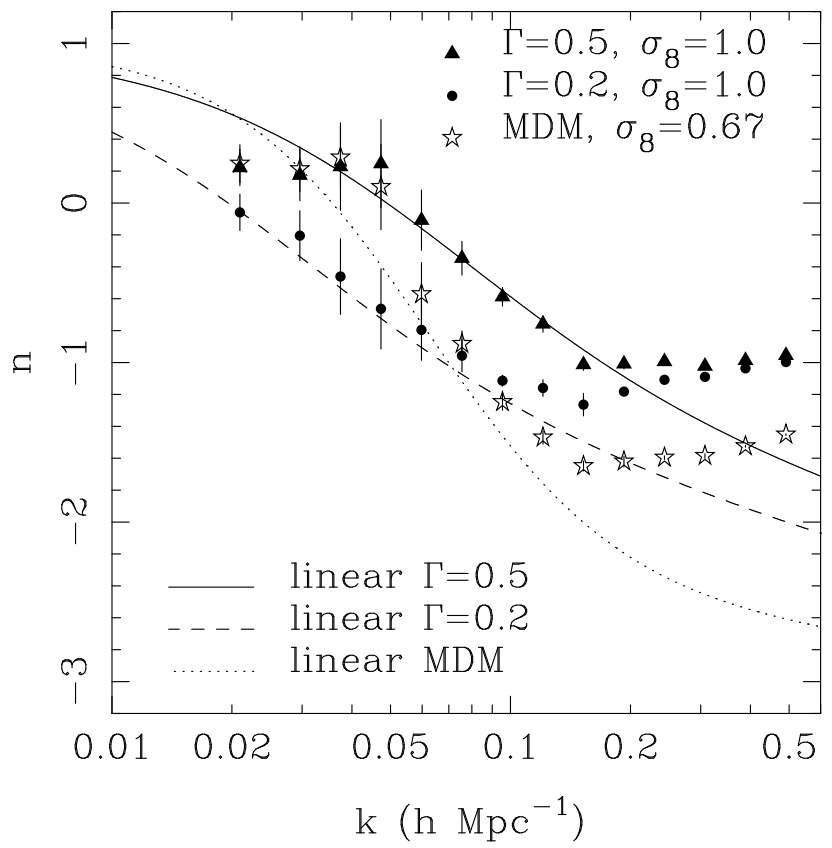

Fig. 5.- The measured non-linear power spectrum logarithmic slope, $n$ for different cosmological models. The points are the mean of results for 5 simulations, with the error on the mean calculated from their standard deviation. The curves are the linear theory values for $n$.

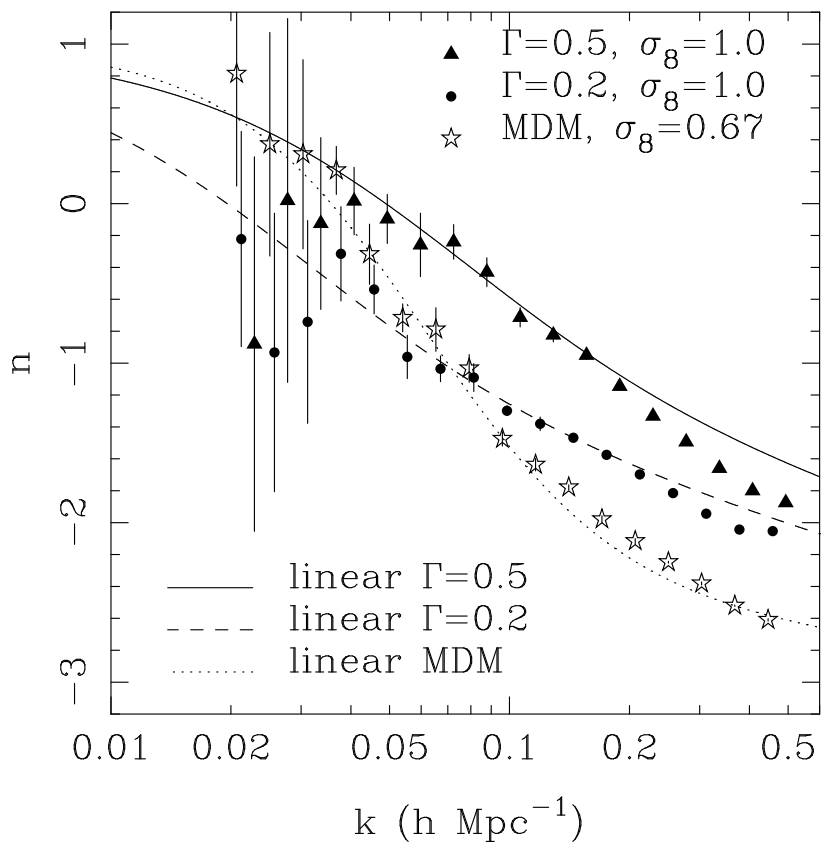

Fig. 6.- Power spectrum logarithmic slope, $n$ reconstructed from the peak space density measured from simulations of three different cosmological models. 


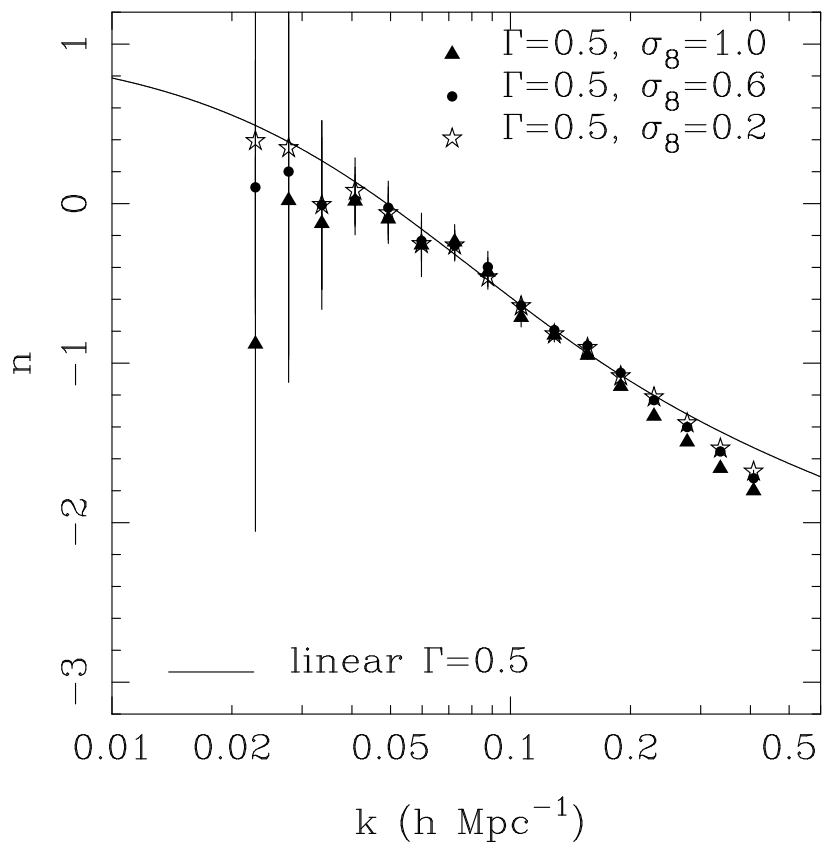

Fig. 7.- Reconstructed power spectrum logarithmic slope, $n$ for three output times of the $\Gamma=0.5$ CDM simulation.

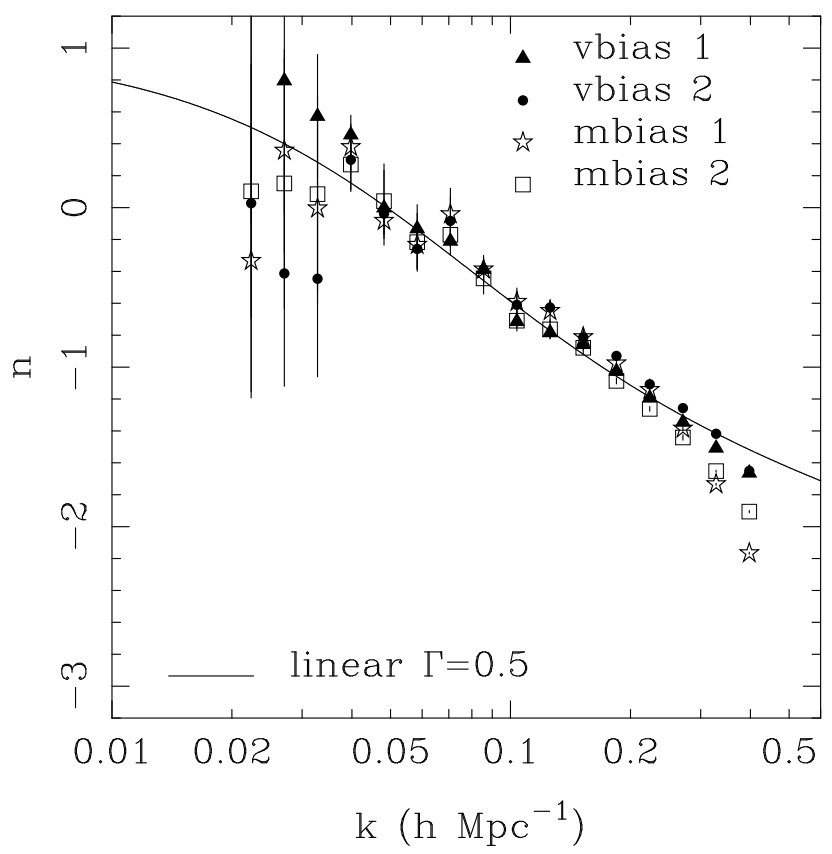

Fig. 8.- Reconstructed power spectrum logarithmic slope, $n$ for the different galaxy biasing prescriptions descibed in Section 2.3 and applied to the $\Gamma=0.5 \mathrm{CDM}$ simulations. 
scales mean that galaxy clustering observations generally favour neither one or the other. We propose that observational measurements of the peak space density would constitute a simple means of telling them apart.

Attempts have already been made to constrain models based on linear power spectra reconstructed from the non-linear observations using mapping formulae calibrated against $N$-body simulations (as mentioned in Section 1). Unfortunately, two different analyses have come up with different conclusions, each favouring a different model, Low density CDM for Peacock \& Dodds (1994) and MDM for Baugh \& Gaztanaga (1996). This may be because of the sensitivity of the non-linear mapping formulae to cosmological parameters such as $\Omega$, or the fact that they work less well with steep power spectra. The reconstruction from the peak space density should not be sensitive to the values of $\Omega$ and $\Lambda$, for example, and should be more insensitive to variations in galaxy biasing. It should be borne in mind, though, that the work in this paper is aimed at the quasilinear regime and that the non-linear mapping formulae can, in principle, be used as a tool to study much smaller scales (although the uncertainties due to biasing could be large).

If one assumes that the initial conditions were Gaussian, we have shown that it is possible to reconstruct the shape of $\mathrm{P}(\mathrm{k})$ on interesting scales from the peak density. The method appears to work best for the models with the steeper slopes on small scales, MDM and $\Gamma=0.2 \mathrm{CDM}$. This

is just as well, as these models are more favoured by galaxy clustering data. The effects of sparse sampling, boundary conditions and redshift distortions must be studied in conjunction with an application to real data. As with the genus statistic, a large contiguous volume is necessary in order to estimate $n_{p k}$ reliably. With the next generation of large redshift surveys (Colless 1997, Gunn \& Weinberg 1995) it should be possible to measure $n_{p k}$ with comparable accuracy to the simulations we have presented here, and probably with even better accuracy on large scales.

In conclusion, we have shown that the space density of peaks in a density field smoothed with a Gaussian filter is independent of time, following the linear theory prediction, even on scales where the clustering is mildly non-linear. We have also shown that the peak space density in itself can be used to distinguish between cosmological models, including the currently popular MDM and Low density CDM scenarios. The peak density could also be used to compare with non-Gaussian models, if predictions become available. We have developed a simple, stable method of recovering the power spectrum from the space density of peaks and demonstrated that the method works using analytic power spectra and the simulation results. An application of the method to a contiguous, densely sampled galaxy survey should yield a useful quantity, the shape of the linear power spectrum on scales $k \lesssim 0.4 h^{-1} \mathrm{Mpc}^{-1}$.

\section{Acknowledgments}

RACC would like to thank CSIC and CESCA(HCM) for financial support and the members of the Institut d'Estudis Espacials de Catalunya for their hospitality. RACC also thanks David 
Weinberg for useful discussions and acknowledges support from NASA Astrophysical Theory Grants NAG5-2864 and NAG5-3111. EG thanks the Astrophysics group in Oxford for their hospitality, and acknowledges support from CSIC, DGICYT (Spain), project PB93-0035 and CIRIT (Generalitat de Catalunya), grant GR94-8001.

\section{REFERENCES}

Bardeen, J. M., Bond, J. R., Kaiser, N., \& Szalay, A. S. 1986, ApJ, , 304,15

Baugh, C. M., Efstathiou, G., 1994, MNRAS, , 270, 183

Baugh, C. M., Gaztañaga, E., 1996, MNRAS, , 280, L37

Bond, J.R., Efstathiou, G., 1984, ApJ, , 285, L45

Colless, M., 1997, in Wide Field Spectroscopy, eds Kontizas M., Kontizas E., Kluwer.

Croft, R.A.C., Gaztañaga, E., 1997, MNRAS, in press, astro-ph/9602100

Dalton, G.B., Croft, R.A.C., Efstathiou, G., Sutherland, W.J., Maddox, S.J., Davis, M., 1994, MNRAS, , 271, 47

da Costa, L., et al., 1997, in Proceedings of the 1996 Texas meeting on Relativistic Astrophysics and Cosmology, eds Olinto,A., Frieman, J. \& Schramm, D.N., World Scientific, Singapore.

Efstathiou, G., Bond, J.R., White, S.D.M., 1992, MNRAS, , 258, 1p

Efstathiou, G., Davis, M., Frenk, C.S., White, S.D.M., 1985, Astrophys. J. Suppl., 57, 241

Gaztañaga, E., Frieman, J.A., 1994, ApJ,437, L13

Gunn, J., Weinberg, D. H., 1995, in Wide-Field Spectroscopy and the Distant Universe, eds S.J. Maddox and A. Aragón-Salamanca, World Scientific, Singapore.

Hamilton, A.J.S., Matthews, A., Kumar, P., Lu, E., 1991, ApJ,, 374, L1

Hinshaw, G., Banday, A.J., Bennett, C.L., Gorski, K.M., Kogut, A., Smoot, G.,F., Wright, E.L., 1996, ApJ, 464, L17

Hockney, R. W., Eastwood, J. W., 1981, Numerical simulations using particles (New York: McGraw-Hill)

Jain, B., Mo, H.J., White, S.D.M., 1995, MNRAS, , 276, L25 
Kaiser, N., 1984, ApJ, , 284, L9

Katz, N., Quinn, T., Gelb, J.M., 1993, MNRAS, , 265, 689

Klypin, A., Holtzman, J, Primack, J., Regos, E., 1993, ApJ, , 416, 1

Lacey, C. G., Cole, S., 1993, MNRAS, , 262, 627

Lucy, L.B., 1974, AJ, 79, 745

Melott, A. L., Weinberg, D. H., Gott, J. R., 1988, ApJ, , 328, 50

Nusser, A., Dekel, A., 1992, ApJ, , 591, 443

Park, C.,1991, MNRAS, , 251, 167

Peacock, J. A., Dodds, S.J., 1994, MNRAS, , 267, 1020p

Peebles, P.J.E., 1980, The Large Scale Structure of the Universe: Princeton University Press

Peebles, P.J.E., 1989, ApJ,, 344, L53

Press, W.H., Schechter, P.H., 1974, 187,425

Weinberg, D. H., 1992, MNRAS, , 254, 315

White, S.D.M., Efstathiou. G., Frenk, C.F. 1993, MNRAS, , 262, 1023 


\section{A. From the peak density to the power spectrum slope}

We will try a simple local reconstruction method which assumes that the shape of $P(k)$ changes slowly with scale. In this case, we can approximate $P(k)$ locally with a power law $P(k)=k^{n}$. For the case of interest, i.e. a density field smoothed with a Gaussian filter the relation between $R_{*}$ and $r_{f}$ for a power law $P(k)$ is given by equation 4 . We have seen in Section 3 that this is only true for all $r_{f}$ if $n$ is a constant. If the shape is slowly varying, a good estimate of $n$ will be the mean local value taken assuming a one to one mapping between values of $k$ and $r_{f}$. We need to find a good effective relation between $k$ and $r_{f}$ in order to use equation 4 to translate the estimated values of $R_{*}$ at a given $r_{f}$ to mean values of $n$ at a given $k$. The form we use is as follows,

$$
k \equiv \eta \frac{\pi}{r_{f}}
$$

where in general $\eta$ may have some dependence on the scale $k$ which will be unimportant if the shape of $P(k)$ varies slowly. For a given family of $P(k)$ the value of $\eta=\eta(k)$ can be found by equating the value of the local slope $n$ at $k$ with the mean value $n$ obtained from equation 4 , after finding the exact relation for $R_{*}=R_{*}\left(r_{f}\right)$. In the case of a simple two power-law model with a break:

$$
P(k)=A k^{n}\left[1+\left(k_{c} / k\right)^{m}\right],
$$

we find that $\eta$ is indeed constant as a function of $k$ :

$$
\eta=\Gamma\left(\frac{5+n}{2}\right) / \Gamma\left(\frac{5+n-m}{2}\right)
$$

and only depends weakly in the shape (but not the position) of the break (at $k_{c}$ ).

In our reconstructions we take $\eta$ to be a constant. We find the best value of $\eta$ for each given $P(k)$ by finding the value which gives the smallest difference between the measured value of $n_{p k}$ and the value predicted from the reconstructed $P(k)$. We test this for a few arbitrary power spectra which we plot in Figure 9. The $\Gamma$ CDM models are taken from Efstathiou, Bond \& White (1992).

In Figure 10 we show, as a function of $\eta$, the rms percentage error per logarithmic bin in the $k$ range corresponding to $R_{f}=2-80 h^{-1} \mathrm{Mpc}$ for: a) the reconstructed value of $(n+5)$ (continuous line), compared to the input slope, and b) the value of the number density $n_{p k}$ calculated from the reconstructed $P(k)$ (dashed-line), compared to the value of $n_{p k}$ calculated directly from $P(k)$.

As expected, the two power-law model (A2) gives zero error at the $\eta$ predicted by (A3). For the other models the error does not approach zero, indicating that the true $\eta$ is not exactly a constant. Nevertheless the minimum rms error value (which defines the best value of $\eta$ ) is typically small. When applying the method to observations, as the true $P(k)$ is not available, we can only estimate $\eta$ by comparing the errors between the observed and predicted $n_{p k}$ (dashed lines in Figure 10), but as shown in the Figure this recovers well the best value of $\eta$ at the minimum rms. 


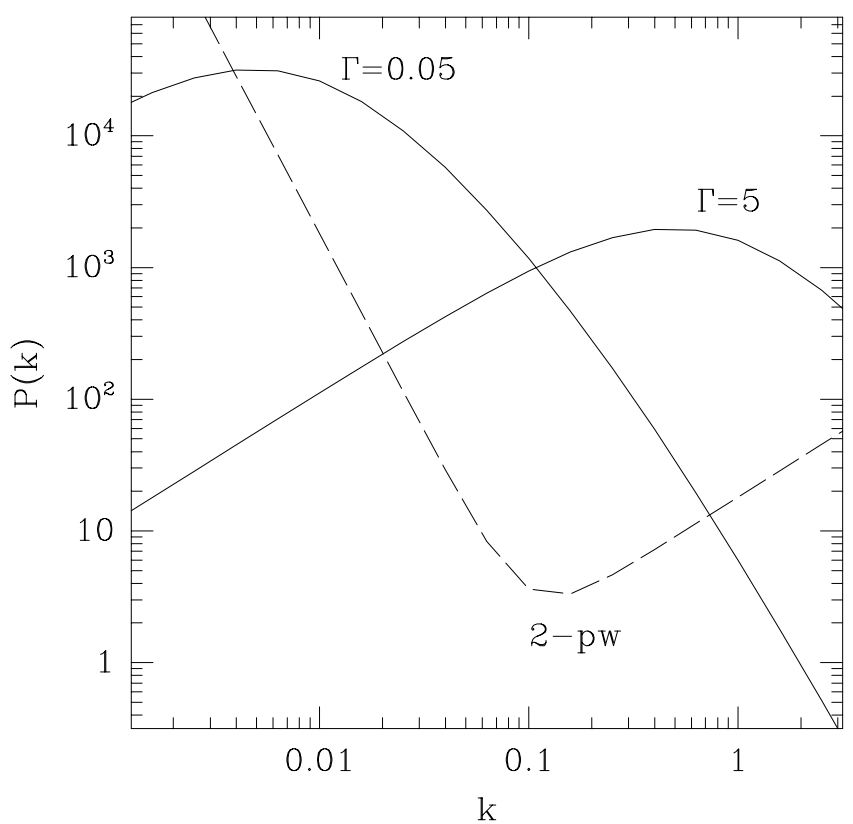

Fig. 9.- Different shapes for $P(k)$ : CDM like models with $\Gamma=0.05$ (continuous left line) and $\Gamma=5.0$ (continuous right line), 2 power-law model (A2) with $n=-3$ and $m=-4$.

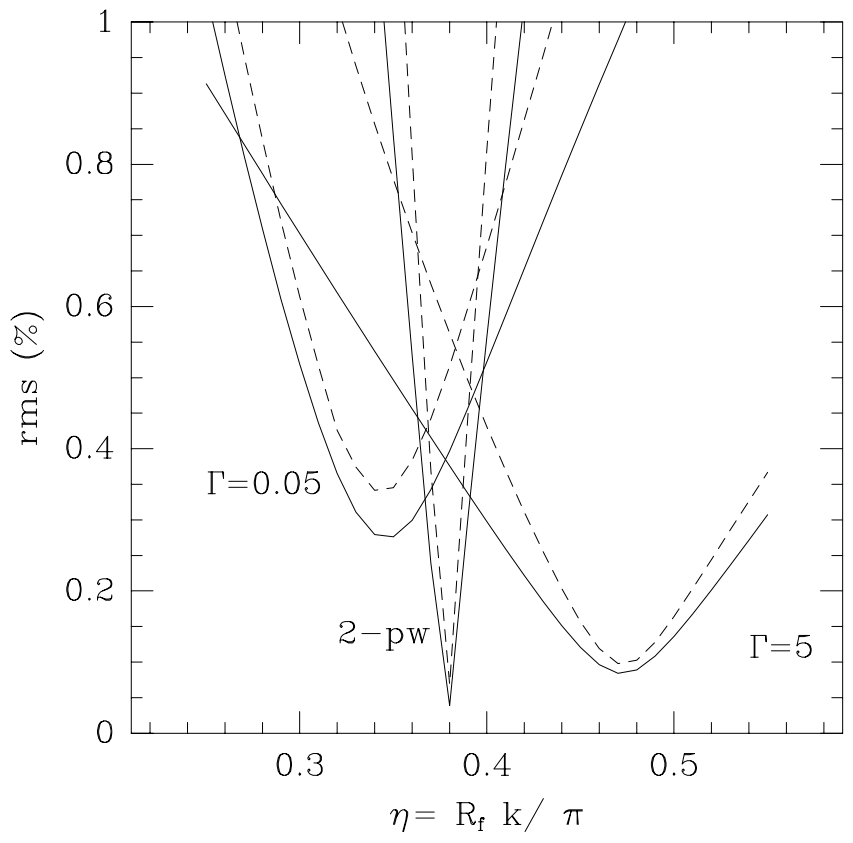

Fig. 10.- For each of the models in Figure 7 we show the percentage rms error per logarithmic bin in: a) $(n+5)$, where $n$ is the logarithmic slope of $P(k)$ (continuous line) b) the number density of peaks (dashed line). 
In Figure 11 we show a comparison of the reconstructed logarithmic slope (3) for the values of $\eta$ that minimise the differences between the input and reconstructed $n_{p k}$. The $k$ range corresponds to $R_{f}=2-80 h^{-1} \mathrm{Mpc}$, which is the input range in $n_{p k}$. The agreement in all cases is quite good (less than $0.5 \%$ rms error per bin).

In Figure 12 we show two ad-hoc example power spectra where the method does not work quite as well as the changes in slope with scale are more rapid. The models shown correspond to power spectra with two sharp breaks or bumps. The rms error in the reconstruction is larger (about $1.5 \%$ rms per bin), but note that the main features, such as the positions and relative amplitudes of the breaks, are still recovered. In these extreme cases a more careful reconstruction method should be used.

Once we have the shape $n=n(k)$, we can of course reconstruct $P(k)$ itself up to an arbitrary constant by carrying out a simple numerical integration. This can be seen in Figure 13 where we show a comparison between the reconstructed and the input $P(k)$ for the values of $\eta$ that minimized the differences between the input and reconstructed $n_{p k}$. To reconstruct $P(k)$ the amplitude has been matched so that $\sigma\left(R_{f}=8\right)=1$ with Gaussian smoothing (we have also rescaled some of the lines in the figure for clarity). As can be seen in this figure, the agreement in all these cases is quite good so that $P(k)$ can be easily recovered, up to a constant. 


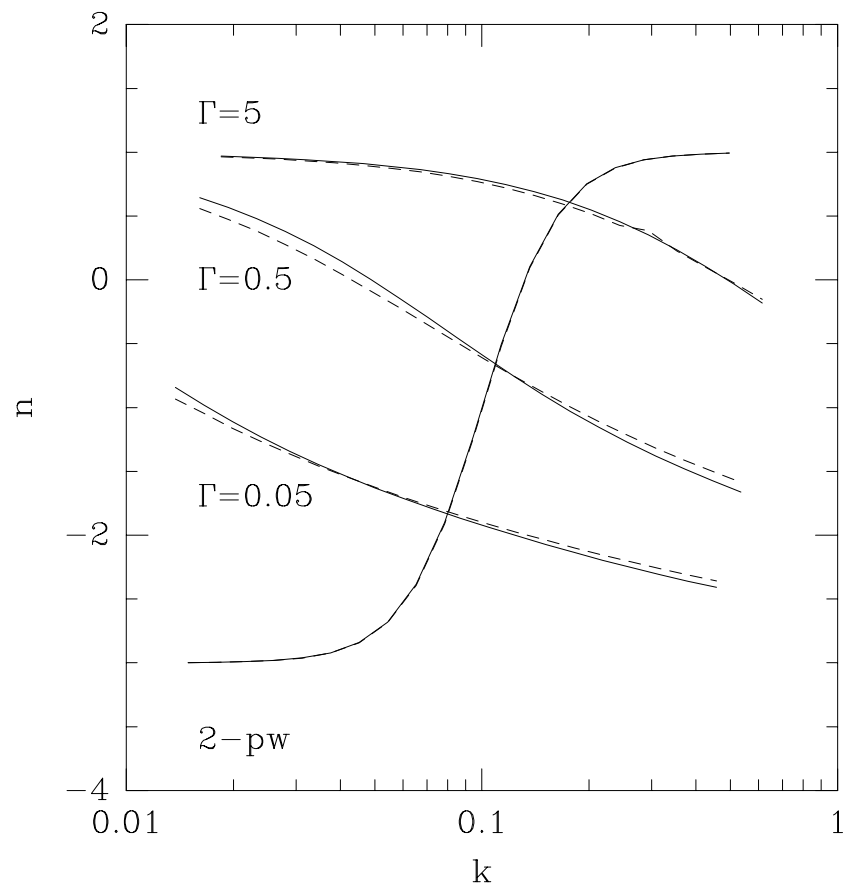

Fig. 11. - The reconstructed (dashed-line) and the original (continuous line) slope of the power spectrum for different CDM like models with $\Gamma=5.0$ (top), $\Gamma=0.5$ and $\Gamma=0.05$. The 2 power-law model (A2) with $n=-3$ and $m=-4$ is also shown.

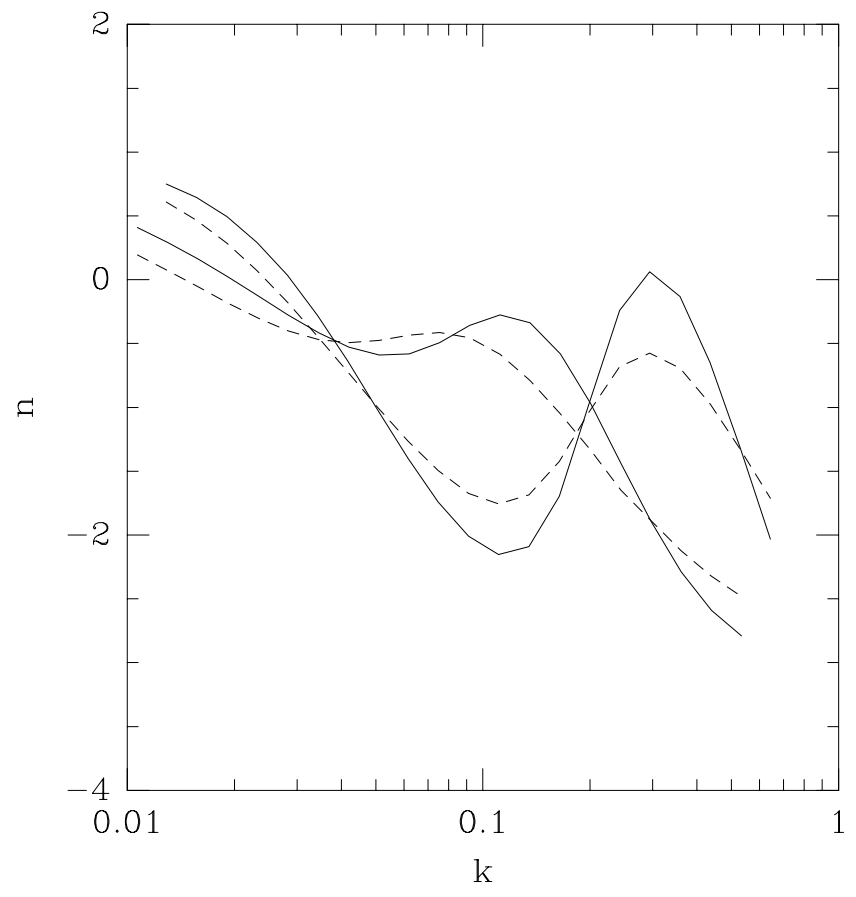

Fig. 12.- The reconstructed (dashed-line) and the original (continuous line) slope of the power spectrum for two models with rapid slope variation. 


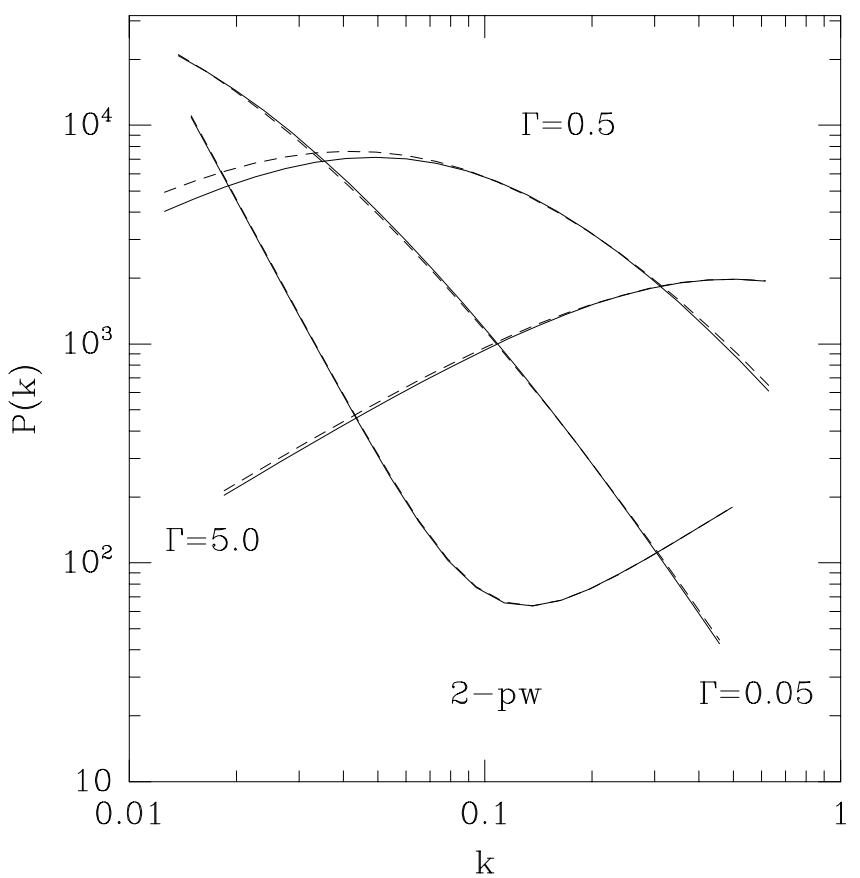

Fig. 13. - For each of the models in Figure 11 we show the reconstructed (dashed-line) and the original (continuous line) power spectrum. 